\title{
Semi-Immersive Space Mission Design and Visualization: Case Study of the "Terrestrial Planet Finder" Mission.
}

\author{
Ken Museth* \\ Alan Barr \\ Computer Science Department \\ California Institute of Technology \\ Pasadena, CA 91125
}

Martin W. Lo ${ }^{\dagger}$

\begin{abstract}
This paper addresses visualization issues of the Terrestrial Planet Finder Mission[2]. The goal of this mission is to search for chemical signatures of life in distant solar systems using five satellites flying in formation to simulate a large telescope. To design and visually verify such a delicate mission one has to analyze and interact with many different 3D spacecraft trajectories, which is often difficult in 2D. We employ a novel trajectory design approach using invariant manifold theory, which is best understood and utilized in an immersive setting. The visualization also addresses multi-scale issues related to the vast differences in distance, velocity, and time at different phases of the mission. Additionally, the parameterization and coordinate frames used for numerical simulations may not be suitable for direct visualization. Relative motion presents a more serious problem where the patterns of the trajectories can only be viewed in particular rotating frames. Some of these problems are greatly relieved by using interactive, animated stereo 3D visualization in a semi-immersive environment such as a Responsive Workbench. Others were solved using standard techniques such as a stratify approach with multiple windows to address the multiscale issues, re-parameterizations of trajectories and associated 2D manifolds and relative motion of the camera to "evoke" the desired patterns.
\end{abstract}

\section{Introduction}

Modern space missions are increasingly more complex as advanced instrument and spacecraft technologies enable more demanding scientific queries during space exploration. Visualization of complex scenarios and high dimensional parameter spaces are extremely useful for the design of such missions. We are developing an interactive, semi-immersive visualization tool called LVis as part of a trajectory design tool (LTool) to support new space missions at the Jet Propulsion Laboratory (JPL). We selected the visualization of the Terrestrial Planet Finder Mission (TPF) as a case study to identify and analyze the requirements for LVis using trajectory data generated in a previous study for NASA[3]. The goal of TPF is to find Earth-like planets around stars near our Sun using interferometry. The mission concept for this case study involves flying a constellation of five satellites in formation around a 3D periodic halo orbit near a so called Lagrange Point ( $L_{2}$ in Fig. 2). For this halo orbit mission we also employ a novel design technique for the low-fuel transfer of the TPF mothership from Earth to the halo orbit. Specifically the transfer trajectory is selected from a family of low-energy trajectories which form a tube-like surface called the stable manifold emanating from the halo orbit. The main contribution of this paper is to demonstrate how this novel approach to low-energy trajectory design can conveniently be analyzed and

\footnotetext{
"kmu@gg.caltech.edu

† Jet Propulsion Laboratory

0-7803-7200-x/01/\$10.00@2001 IEEE.
}

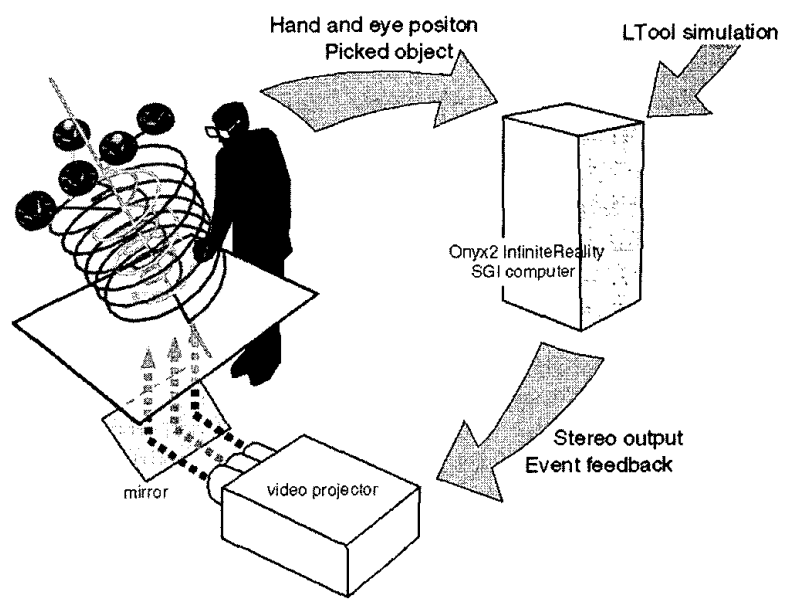

Figure 1: Illustration of the Responsive Workbench at Caltech.

understood using techniques such as animated non-immersive and interactive immersive visualization.

The paper is organized as follows: Section 2 presents an overview of the scientific goals of the TPF mission, its different phases, and briefly the model for the simulation of the trajectories. Section 3 gives an outline of the requirements, problems and solutions for the TPF visualization. Section 3.1 discusses the multiscaling problems and a simple stratify solution. Problems and solutions involving re-parameterization of trajectories and manifolds for visualization are described in Section 3.2. The semi-immersive interaction with the trajectories and manifold is presented in Section 3.3, and a non-immersive animation system is presented in Section 3.4. Finally Section 3.5 discusses some implementation issues, and Section 4 concludes and mentions plans for future work.

\section{The Terrestrial Planet Finder Mission}

The TPF Mission[2], which is scheduled for launch 2012, is one of the centerpieces of the NASA Origins Program. The goal of TPF is to identify Earth-like planets around stars nearby our Sun. For this purpose, a space-based infrared interferometer with a baseline of approximately $100 \mathrm{~m}$ is required. To achieve such a large interferometry baseline, a distributed system of five satellites flying in formation is an efficient approach. The current concept has four 3.5 $m$ diameter telescopes, each with its own propulsion system, and a central collector satellite that combines the four beams. Since the TPF instruments need a cold and stable environment, near-Earth orbits are unsuitable. An ideal solution is to place TPF in a halo orbit as shown in Fig. 2. The dynamics of this halo orbit mission dictate the requirements for the visualization to be described in the next section. 


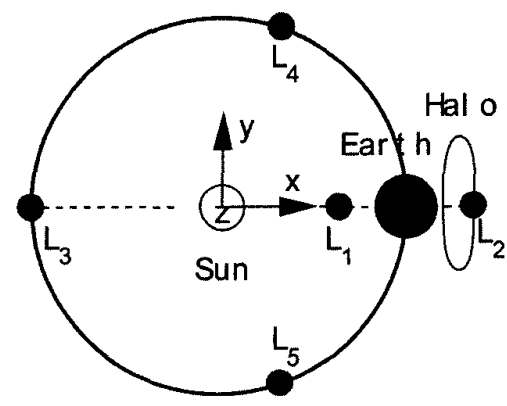

Figure 2: The Lagrange points are five equilibrium (i.e. zero total force) points in the orbit of one body around another, such as the Earth around the Sun. $L_{1}, L_{2}$, and $L_{3}$ are unstable with respect to small perturbations, whereas $L_{4}$ and $L_{5}$ are stable equilibrium points. The halo orbital considered for the TPF mission is a 3D periodic orbit around the $L_{2}$ Lagrange point.

\subsection{The Model for the TPF Simulation}

We model the dynamics of TPF in the Circular Restricted Three Body Problem (CRTBP) where the Sun and the Earth are moving in circular orbits around the center of mass, and the third body (a massless spacecraft) moves under their influence in the XY-plane [3]. The most natural frame to use in the CRTBP is a rotating frame with the origin at the center of mass, the $\mathrm{X}$-axis along the Sun-Earth line, and the Earth on the positive X-axis, as shown in Fig. 2.

$L_{2}$ is one of the five Lagrange points. It lies on the far side of the Earth along the Sun-Earth line as depicted in Fig. 2. A halo orbit is a 3D, large, unstable periodic orbit around $L_{2}$ with a shape simular to the edge of a potato chip. The instability of the halo orbit is very small (time constant on the order of a month) and it provides a whole family of low energy transfer trajectories from the Earth to the halo, forming a tube called the stable manifold of the halo orbit. Fig. 4(b) on the color plate depicts a portion of the stable manifold (green) with a family of transfer trajectories (red) from the Earth to the halo orbit. The trajectories and the 2D manifold are actually objects in the 6D phase space composed of position and velocity. We are visualizing the projection of these objects into 3D position space. In fact, if we continued the computation of the stable manifold, the visualization of its projection in position space will show self-intersections.

Astrodynamicists have determined how to break a trajectory into blocks that may be designed somewhat independently. These blocks can be assembled in tinker-toy fashion, following rules and constraints, to produce an end-to-end trajectory. The gluing of these blocks is an art and typically uses multiple shooting algorithms or two-point-boundary value methods[3]. For halo orbit missions, the blocks are the launch orbit, the parking orbit, the transfer orbit, and the halo orbit.

\subsection{Low-Energy Transfer to Halo Orbit}

For this study, we assume that we already have the halo orbit and its stable manifold (see [3] for details). The designer next selects an insertion point on one of the red transfer orbits (color plate Fig. 4(a) and 4(b)) embedded on the stable manifold with the picking device. During this step LVis provides useful information to the designer, like fuel consumption to reach the halo orbit, velocity (red arrow) etc., that will help him in this decision process. LVis then computes a circular parking orbit around the Earth that connects to the insertion point on the selected white trajectory on the manifold. We do not compute and visualize the launch orbit from Earth to the parking orbit because this is considered a separate problem that does no have to be considered during the early study phase of the mission.

\subsection{Formation Flight Around Halo Orbit}

Once an end-to-end trajectory from a parking orbit to the halo orbit has been designed, we move to the next phase of the simulation, the Observation Phase. We assume the spacecraft carrying the five satellites for the TPF mission is now on the halo orbit. At a predetermined moment, the five satellites are deployed from the spacecraft and maneuver into the initial formation where four of the satellites are equally spaced on the diameter of a 100 meter spinning circle, and the fifth satellite (the beam combiner) is offset from the center of the circle by 50 meters. The observations of the four satellites are beamed to the collector satellite. The spin of the circle synthetically creates a telescope with a $100 \mathrm{~m}$ diameter. For this simulation, the satellites are actually moving along a 20 sided polygon using small impulsive rocket burns at the corners of the 20-gon. These impulsive burns also include corrections due to the small instability of the halo orbit and gravitational perturbations from other planets. The angular momentum vector of the spinning circle point at the star that TPF is currently observing. After making several revolutions in the direction of one star, TPF reorients the spinning 20 -gon by reconfiguring the satellites into the next spinning 20-gon pointing at the next star to be observed. Fig. 4 (c) on the color plate shows the spirals generated by the TPF formation as it makes its observation along the halo orbit (straight blue orbit across the image).

\section{Visualization}

The TPF mission design strategies presented in the previous section form a complex problem that may be studied with an interactive simulation environment that offers constant visual feedback. The goals and requirements of the graphics tool for the TPF simulation presented in this paper can be broken into the following steps.

1. Animate the time-propagation of the stable manifold of the halo orbit associated with the $L_{2}$ Lagrange points of the SunEarth system.

2. Select a low-energy transfer trajectory on the stable manifold and compute a conic low-Earth parking orbit for the spacecraft that intersects the manifold trajectory at the selected insertion point.

3. Launch the spacecraft with TPF satellites from the intersection point of the parking orbit along the low-energy trajectory onto the halo orbit.

4. Once the spacecraft reaches the halo orbit, deploy the five satellites into initial formation.

5. Begin satellite pattern maintenance on a 20 -gon by making impulsive rocket burns at the vertices to form a large virtual telescope.

6. Satellites make reconfiguration maneuvers to reorient the line of sight by tilting the plane of the 20 -gon.

This requirements list for $L V i s$, the interactive design and visualization tool of the TPF mission, presents a number of graphical problems which is the topic of the remaining of this section.

\subsection{Multi-Scaling Issues}

A problem often encountered when visualizing astronomical data is the enormous differences in scale that they typically represent. The differences can be both in spatial distances, time lines for events and relative velocities to some fixed frame of reference. This is especially pronounced in the present case study of the TPF mission. For instance, the distance from Earth to $L_{2}$ is 1.5 million $\mathrm{km}$ and the baseline halo orbit has amplitudes on the order of $700,000 \mathrm{~km}$ whereas the formation flight of the five satellites around it has to be 
accurate to within 2 centimeters. Also the velocity of the satellites relative to each other is typically a few $\mathrm{m} /$ hour whereas their relative speed to the halo orbit is several thousand $\mathrm{km} / \mathrm{hour}$. The transfer of the spacecraft from a parking orbit around Earth, along a lowenergy transfer trajectory, onto the halo orbit takes several months. In contrast, the pattern formation of the satellites typically spans a few hours. These enormous differences in scales (a factor of $10^{13}$ for distance) clearly cause problems both for the visualization as well as the numerical simulation. The latter is conveniently solved by partitioning the numerical simulation of the mission into two phases: a computation of the stable manifold in a Sun-Earth rotating frame, and the computation of the satellite motion in a moving frame relative to the baseline halo orbit. This simple but effective strategy suggests a similar stratified approach to the visualization. Thus, the design and visualization of the mission is rendered in two separate windows on the monitor or as two different applications on the RWB - one for the transfer from Earth to the halo orbit on a scale of astronomical units $(149,600,000 \mathrm{~km})$ and a time-scale of days (step 1 through 3 in the list in Section 3) - and one for the formation flight on a scale of meters and minutes (step 4-6). However, one adjustment had to be made to this approach; since the formation flight is simulated in a frame that moves with a velocity equal to the halo baseline this gave a misleading visual illusion of the satellites flying in static periodic orbits around the baseline. To fix this we introduced a new frame of reference in which the camera is fixed, but the satellites move slowly forward and the spacecraft slowly backward. This enables the visualization of the pattern of the satellite formation.

\subsection{Parameterization Issues}

A fundamental problem when rendering the trajectories is the parameterization. For the numerical simulation a natural parameterization of the trajectories is by time. Specifically the sampled representations of all the trajectories were given by fixed timeincrements. However, due to the rather complex nature of the family of trajectories embedded on the stable manifold in $6 \mathrm{D}$ phasespace, such a parameterization is not suitable for visualization see Fig. 3. Since the velocity on the trajectory changes dramati-
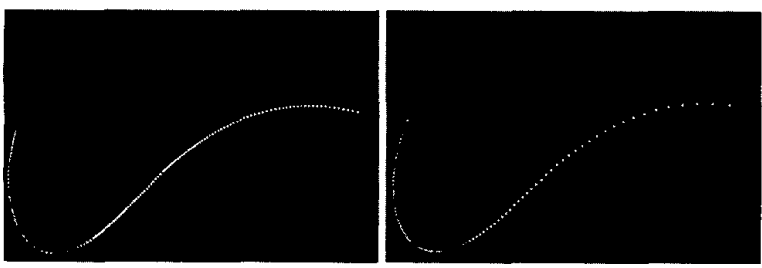

Figure 3: Arclength parameterized orbit antialiases the rendering to produce a smooth curve (left), time parameterized does not (right).

cally in space, a fixed time parameterization produces very jagged or aliased lines when the trajectory is projected to the 3D position space. On the other hand a fixed arclength parameterization would produce many redundant sampling points in regions near the halo orbit where the velocity is very low. Thus, we developed a simple scheme for the arclength parameterization of the manifold trajectories that preserves as many of the original data points as possible. Using the three coordinate and three velocity components at each pair of two adjacent original sampling points in the $6 \mathrm{D}$ phase-space, $\left(x\left(t_{1}\right), v\left(t_{1}\right), x\left(t_{2}\right), v\left(t_{2}\right)\right)$, a cubic polynomial is analytically fit to the corresponding curve segment. If the arclength $s\left(t_{1}, t_{2}\right)=\int_{t_{1}}^{t_{2}}|v(t)| d t$ is smaller than a given minimum arclength the point at $t_{2}$ is removed and if it is integer $n$ times larger than a given maximum arclength new sampling points are inserted at times $\left.t_{i}^{\prime} \in\right] t_{1}, t_{2}[, i=1, . ., n$ such that $s\left(t_{1}, t_{i}^{\prime}\right) / s\left(t_{1}, t_{2}\right)=i /(n+1)$. The times $t_{i}^{\prime}$ are numerically found by a Newton-Raphson iteration as described in reference [7] and the bounds on the arclength are chosen to produce sufficiently smooth trajectories. This subdivision algorithm proved efficient for the reparameterization of the manifold trajectories (see Fig. 3), and additionally generates important information about the accumulated arclength along each of the transfer trajectories that is returned to the user upon selections in step 2 of the list above. However, a different approach had to be employed for the triangulation of the manifold tube. The growth of the manifold has to be animated as time evolves requireing a time parameterization that ensures a welldefined tube-like geometry for the manifold during all stages of the animation. Furthermore, the subdivision scheme outlined above generates a different number of sampling points for each manifold trajectory. This prevents the creation of a simple triangulation with rings of triangle strips from sets of two connecting points on each trajectory. On the other hand the original time-parameterized points undersample the tube in regions of space with large velocity (typically near Earth) producing bad aspect-ratio triangles. A robust solution was simply a finer re-sampling of the trajectories in time.

\subsection{Semi-Immersive Visualization}

We found it extremely useful for this study to employ the Responsive Workbench (RWB) at Caltech (see Fig. 1). This is a 3D interactive virtual reality system with a tabletop metaphor originally developed by Wolfgang Krueger at GMD[6]. The user of the RWB sees the virtual objects as floating over the tabletop and can interact with them just as they would with real objects on an actual workbench. This allows for the mission designer to quickly develop a perception of the 3D structure of the stable manifold which in turn is very valuable both for general analysis, as well as the selection of initial conditions for the transfer trajectory embedded on the manifold (step 2 in the list above). To create this 3D environment, users wear shutter glasses (V-sync at $120 \mathrm{~Hz}$ ) to view computer-generated stereoscopic images that are projected on the tabletop display surface by a projector/mirror system. The user's head position and orientation are tracked to create the correct perspective for the computer to use when rendering the environment. A 6DOF input device from Polhemus is also tracked by the system allowing the users to accurately interact with the trajectories, see Fig. 1. We especially found the stereoscopic feature of the RWB to be very useful for this study primarily because we are analyzing unfamiliar trajectories with complicate 3D structures. The immersive interaction with the data also seems more natural on the RWB than on a $2 \mathrm{D}$ monitor where picking is typically implemented by ray intersection of bounding boxes and can lead to ambiguous selections such as points on either side of the manifold tube.

\subsection{Animation Systems}

Physically-based animation[7] is naturally implemented using a procedural approach where the simulation explicitly defines the movements of objects as a function of time. However, to allow for the animation to include objects that were not explicitly included in the actual calculations, (e.g. rotating stars and moon) and to properly synchronize events and phasing of the mission like the growth of the manifold and launch time, we also implemented an eventdriving scripting system. This allows the user to completely control the sequence of events and to "play" the mission forward or backward at any desired speed.

To conveniently communicate a completed mission design by an automated animation or to generate video animations a 3D key frame animation system was also implemented. It allows the user to interactively select the camera position and orientation at different 
times of the mission, which are then played back by interpolating the key frames. The camera orientation is explicitly represented by quaternions and the interpolation is done as three successive spherical cubic interpolations as explained in reference [4]. The system then performs off-screen rendering of the scene and dumps the frame-buffer to image files.

\subsection{Implementations And Visual Clues}

An important requirement for $L V$ is is that it should be interactive on a non-immersive desktop workstation for fast prototyping, and also flexible enough to be ported to a semi-immersive environment on high-end SGI hardware. The real-time requirement constrained the graphics to only fiat or Gouraud-shaded polygons with texture mapping, and the portability issues lead us to select the open-sourced OpenInventor API[5].

As emphasized in Section 3.1 it was numerically convenient to stratify the visualization of the TPF mission into two phases or scene graphs - the transfer followed by the formation flight. Common to both scene graphs was a background star-map implemented by projecting the true ephemeris on a rotating sphere placed at the center of the bounding-box. Both scenes also have a directional light source oriented according to the position of the Sun in order to obtain the correct lighting of all objects.

The "transfer-scene-graph", Fig. 4(b), has a grid in the XY-plane of the rotating Sun-Earth frame and texture-maps of the spinning Earth with the Moon in a conic orbit around it. The direction of the Sun is indicated by a glowing yellow arrow and the static position of $L_{2}$ by a simple colored sphere placed on the Sun-Earth X-axis. The scene also includes a texture-mapped spacecraft with five satellites orbiting around Earth in a conic orbit which by construction intersects the user-selected transfer trajectory. The halo orbit is shown as a closed curve around $L_{2}$, and the stable manifold is grown as a transparent tube and has the embedded family of trajectories twisting around it. After the transfer trajectory is highlighted and the corresponding parking orbit is computed from a selected insertion point the spacecraft makes a small burn and flies on the stable manifold onto the halo orbit.

The "formation-scene-graph", Fig. 4(c), shows a very small segment of the halo orbit with the spacecraft and five satellites attached to it. For this visualization, we implemented a number of visual and audio cues to emphasize the rather complex dynamics. As already mentioned the illustration of motion relative to the halo orbit was artificially introduced, and to enhance this the five spiraling trajectories of the satellites are dynamically rendered with color coding. To emphasize the detailed relative motion of the satellites a transparent 20-gon is added to the scene and the orientation of the virtual telescope is illustrated by an arrow pointing in the line of sight of the IR interferometer. The rocket burns of the satellites at each vertex of the 20-gon are emphasized by short pulsating animations of fire accompanied by a sound effect.

\section{Conclusions And Future Work}

We have presented an interactive design, visualization, and animation tool, LVis, for the TPF mission. Our investigations and prototypes show the RWB metaphor to be very useful for visualizing complex geometries. The RWB is able to provide 3D visual cues and nuances absent in non-immersive environments, which is particularly important for this study since most of the trajectories are new and unfamiliar to mission designers. In the case of the lowenergy transfer from Earth to the halo orbit we employ a novel design strategy that produces unfamiliar trajectories embedded on complicated 3D manifolds. In the case of the trajectories spinning around the halo orbit, this is the first demonstration that such tight formation flight is possible. Hence, it is even more important to provide additional insight through immersive visualization to help the aerospace and scientific communities understand this new design approach.

We solved the multi-scale problems using stratification approach with multiple windows. We solved the aliasing problems by reparameterizing the orbits by arclength and by re-sampling the manifolds in time. We solve the manifold animation problem by propagating the manifold tube in annular strips which are dynamically triangulated. We solved the problem of visualizing the formation pattern by moving the camera in a proper manner. We provided picking on the trajectories and manifolds to enable intuitive and convenient exploration of large data sets. We provided a scripting language to create efficient $3 \mathrm{D}$ key-frame animations using quaternions.

We plan to conduct a detailed user analysis of our visualization and design tool in the near future when it has further matured. One of the improvements that we would like to see is a different solution to the multi-scale problem that will allow all phases of the mission design to be handled in the same window/application. For this we are currently investigating a camera model by Blinn[1] which allows for the specification of spacecraft and planets as respectively foreground and background objects in the same scene. Other plans for future work include the computation and visualization of intersections of different manifolds to provide new options for low energy trajectory design. We plan to study the use of haptic devices to add another dimension to the immersive experience. We are also exploring the option of providing semi-immersive capabilities on a desktop workstation as a low-cost alternative to the RWB.

\section{Acknowledgment}

This work was carried out at the Jet Propulsion Laboratory and the California Institute of Technology under a contract with National Aeronautics and Space Administration. The work was partially supported by the National Science Foundation (ASC-89-20219 and ACI-9982273). We thank Josep Masdemont and Gerald Gómez for the TPF simulation performed in a previous collaboration[3]. Finally we thank David Breen for many useful comments and Mark Montague for assisting the video production with this paper.

\section{References}

[1] J. Blinn. Where Am I? What Am I Looking At? IEEE Computer Graphics And Applications, 8(4):76-81, July 1988.

[2] Beichman C.A., Woolf N.J., and Lindensmith C.A. The terrestrial planet finder: A NASA origins program to search for habitable planets. JPL Publication 99-3, May 1999.

[3] G. Gomez, M. Lo, J. Masdemont, and K. Museth. Simulation of formation flight near Lagrange points for the TPF mission. In Proceedings of Astrodynamics Specialist Conference, Quebec City, Canada, 2001. American Astronautical Society.

[4] K. Shoemake. Quaternion calculus and fast animation. In Course Notes, volume 10, pages 101-21. SIGGRAPH, 1987.

[5] P. S. Strauss and R. Carey. An object-oriented 3D graphics toolkit. In Computer Graphics, volume 26, pages 341-349. SIGGRAPH, 1992.

[6] Krueger W. and Froehlich B. The responsive workbench. IEEE Computer Graphics and Applications, 14(3):12-15, May 1994.

[7] A. Watt and M. Watt. Advanced Animation and Rendering Techniques, chapter 15. Addison-Wesley, 1992. 